EPJ Web of Conferences 41, 07010 (2013)

DOI: $10.1051 /$ epjconf/20134107010

(C) Owned by the authors, published by EDP Sciences, 2013

\title{
Ultrafast geminate electron-radical recombination dynamics in photoactive yellow protein
}

Jingyi Zhu' ${ }^{1}$, Laura Paparelli ${ }^{1}$, Marijke Hospes ${ }^{2}$, Jos Arents ${ }^{2}$, Klaas Hellingwerf ${ }^{2}$, John T. M. Kennis ${ }^{1}$, Ivo H.M. van Stokkum ${ }^{1}$, Marie Louise Groot ${ }^{1}$ *

${ }^{1}$ Department of Physics and Astronomy, Faculty of Sciences, VU University, De Boelelaan 1081, 1081 HV Amsterdam, The Netherlands

${ }^{2}$ Laboratory for Microbiology, Swammerdam Institute for Life Sciences, University of Amsterdam, Nieuwe Achtergracht 166, 1010 WV Amsterdam, The Netherlands

\begin{abstract}
Photoinduced ionization of the chromophore inside photoactive yellow protein (PYP) was investigated by ultrafast spectroscopy in the visible-near infrared spectral region. A solvated electron absorption-like band was observed that extended from around $550 \mathrm{~nm}$ to $850 \mathrm{~nm}$, centred at $700 \mathrm{~nm}$. Simulation of the decay traces of the band with a classic diffusion model indicated that ejected electrons were located an average distance of $\sim 3 \AA$ away from the radical centre, and around $40 \%$ the solvated electrons were annihilated by geminate recombination. The remaining $60 \%$ escaped out of the protein pocket to be annihilated by bulk recombination. This result indicates that the chromophore is in a local environment inside PYP that is only slightly different from the bath solvent.
\end{abstract}

\section{Introduction:}

Photoactive yellow protein (PYP) is a blue-light photoreceptor responsible for the negative phototaxis of the bacterium Halorhodospira halophila.[1] The functional chromophore in the protein is the deprotonated trans-p-hydoxycinnamic acid ( $\mathrm{pCa}$ ) anion, which is covalently linked to the side chain of a cysteine residue via a thioester bond. Upon absorption of blue light, the chromophore undergoes trans to cis isomerization around its $\mathrm{C}_{7}=\mathrm{C}_{8}$ bond, marking the beginning of a photocycle and the formation of a signaling state that involves partial unfolding of the protein. The initial photoreaction is complicated by observation of three excited states and the branching into an inactive channel to a ground state intermediate where it returns back to the ground-state.[2] In addition, an ionization channel of the chromophore was also observed in the visible wavelength region, which makes the photoreaction of PYP more complicated. [2] Due to the overlap of the absorption bands of the electron with that of the isomerized product, the character of the ionized electron band and its

This is an Open Access article distributed under the terms of the Creative Commons Attribution License 2.0, which permits unrestricted use, distribution, and reproduction in any medium, provided the original work is properly cited. 
recombination dynamics have not been characterized. In this study we focus our attention on the ionization of the chromophore in PYP and the dynamics of electron-radical recombination. We report for the first time the whole band shape of the ionized electrons and its decay dynamics inside the wild type PYP. To extract the electron dynamics, global target fitting based on kinetic models was applied. Simulation of the recombination dynamics based on diffusion theory was used to understand more details of the ionized electron dynamics and the charge distribution inside PYP.
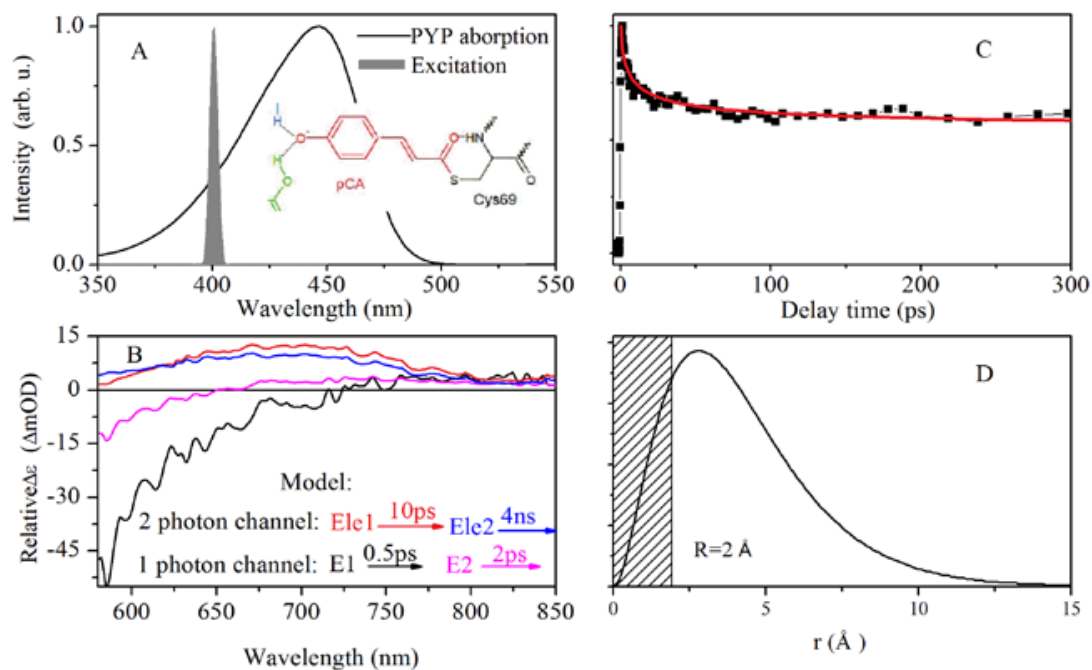

Fig. 1. Spectroscopy of PYP and electron radical recombination dynamics. (A) Steady state absorption of PYP and pump wavelength. Inserted is a structure of the chromophore at the active site. (B) Species associated spectra and target model. (c) Simulated dynamics of electron decay at $700 \mathrm{~nm}$ (red line), and experimental data (solid squares). (d) Simulation parameters and initial distribution of the ionized electron. $3 \mathrm{~b} \sim 3 \AA$, and $\mathrm{D} \sim 0.8 \times 10^{-4} \mathrm{~cm}^{2} / \mathrm{s}$.

\section{Results and discussion:}

Figure 1(A) presents the steady-state absorption of wild type PYP. The excitation pulse is shown as the gray area. At sufficiently high energy, a two photon absorption process will result in ionization of the chromophore, yielding a 'free' electron. The insert shows part of the chromophore structure at the active site in PYP. The absorption changes in the $550-850 \mathrm{~nm}$ region, induced by the excitation of the chromophore, showed rapidly decaying contributions of the stimulated emission of the chromophore and broad induced absorption bands. The absorption changes were globally fitted [3] with a target model incorporating these components, illustrated in Figure 1(B). The fitted Species Associated Spectra (SADS) are shown with the same colour coding as in the model. The first branch has a two-stage decay, the SADS show induced absorption bands, and are due to the two photon ionization channel (2-photon power dependence not shown). The second branch has also a two-stage decay, follows a 1-photon power dependence (not shown) and its SADS show excited state stimulated emission, of which the tail extends to this region. The separation between the one and two photon channels yields very reasonable spectra and is thus considered to be quite good. The decay time constants of the stimulated emission of the one photon excited states are $500 \mathrm{fs}$ and $2 \mathrm{ps}$, consistent with previous observations in the visible region. [2] The first electron band has a lifetime around 12ps and decays to the second band which has a lifetime of around 4ns. Both of the electron bands have similar broad shapes.

For simplicity, we ignore the weak interaction between the ionized electron and neutral radical. Thus, similar as in solvents, a classic model based on Fick's second law of diffusion can be used to reproduce the geminate recombination dynamics in PYP. Assuming an exponential initial 
distribution of the ionized electron in space, the solution to the free diffusion equation is given [4,5] by

$$
C(t)=\int_{R}^{\infty} \frac{\exp (-r / b)}{8 \pi b^{3}} \times\left[1-\frac{R}{r} \operatorname{erfc}\left(\frac{r-R}{\sqrt{4 D t}}\right)\right] \times 4 \pi r^{2} d r
$$

where $\mathrm{D}$ is the sum of the diffusion coefficients of the electron and the radical chromophore, $\mathrm{R}$ is the recombination radius where the electron-radical recombination react immediately, and $\mathrm{C}(\mathrm{r}, \mathrm{t})$ represents the observable ionized electrons not recombined with the radical. The initial spatial distribution of the ionized electrons has an average distance of $3 \mathrm{~b}$ from the centre of the radical.

Using formula (1), the recombination dynamics can be simulated by fitting to the experimental data as shown in figure $1(\mathrm{C})$ at $700 \mathrm{~nm}$. We fixed the constant $\mathrm{R}$ to $2 \AA$, the radius of the negative charged phenolate oxygen atom. The simulated diffusion rate of the solvated electrons is $0.8 \times 10^{-}$ ${ }^{4} \mathrm{~cm}^{2} / \mathrm{s}$, which is close to the one measured in water $0.5 \times 10^{-4} \mathrm{~cm}^{2} / \mathrm{s}$ [6]. Although the crystal structure of PYP shows that only one water (water 200) [7] is buried inside the protein, molecular dynamics simulations in wild type and mutants PYP [8] indicate that about 3 water molecules are located near the phenolate oxygen of the chromophore. The simulated D here suggests that although deeply buried inside PYP, the chromophore is in a local environment that is only slightly different from the bulk water solvent. The simulated decay and solvated electrons initial distribution around the radical are shown in figure $1(\mathrm{C}$ and $\mathrm{D})$. The electron is ejected into the vicinity of the phenolate oxygen of the chromophore, with only a small average distance of $\sim 3 \AA$.

\section{References:}

1. K. J. Hellingwerf, J. Hendriks and T. Gensch, J. Phys. Chem. A, 107, 1082(2003).

2. D. S. Larsen, Ivo H. M. van Stokkum, M. Vengris, M. A. van der Horst, F. L. de Weerd, K. J. Hellingwerf, and R. van Grondelle, Biophys J., 87,1858(2004).

3. I. H.M. van Stokkum, D. S. Larsen, R. van Grondelle, Biochim Biophys Acta, 1657 82(2004).

4. F. C. Collins and G. E. Kimball, J. Colloid Sci. 4, 425 (1949).

5. J. A. Kloepfer, V. H. Vilchiz, V. A. Lenchenkov, A. C. Germaine, and S. E. Bradforth, J. Chem. Phys. 113, 6288 (2000).

6. K. H. Schmidt, P. Han, and D. M. Bartels, J. Phys. Chem., 96, 199(1992).

7. H. Kandori, T. Iwata, J. Hendriks, A. Maeda, and K. J. Hellingwerf, Biochemistry, 39, 7902(2000).

8. A. B. Rupenyan, J. Vreede, I. H. M. van Stokkum, M.Hospes, J. T. M. Kennis, K. J. Hellingwerf, and M. L. Groot, J. Phys. Chem. B , 115, 6668(2011). 